\title{
ANÁLISIS Y DIFUSIÓN DE REACONDICIONAMIENTOS ENERGÉTICOS RESIDENCIALES BASADOS EN SIMULACIONES
}

\section{ANALYSIS AND DISSEMINATION OF RESIDENTIAL ENERGY RETROFITS BASED ON SIMULATIONS}

\author{
Rodrigo García Alvarado ${ }^{1}$ \\ Pedro G. Campos ${ }^{2}$
}

\begin{abstract}
RESUMEN
Este trabajo expone un prototipo computacional para la definición y divulgación de soluciones de mejoramiento ambiental de viviendas unifamiliares en la zona metropolitana de Concepción, Chile. Las viviendas generan un alto consumo energético, por lo que diversos programas públicos e iniciativas privadas permiten adecuar las construcciones existentes para mejorar sus desempeños, con soluciones específicas que se pueden determinar mediante simulaciones dinámicas. Se plantea entonces una herramienta profesional para revisar alternativas, con cálculos de precios y resultados de simulación, para conformar paquetes de mejoramientos por caso. También, este software permite realizar estimaciones según datos básicos, en base a viviendas previamente estudiadas en la zona, para la difusión pública de posibilidades de reacondicionamiento. Se exponen los antecedentes y casos estudiados, así como el desarrollo y funcionalidad del sistema propuesto, actualmente en etapa de prototipo avanzado, y el análisis de algunos casos. El software permite determinar paquetes de reacondicionamiento que generan reducciones del $35 \%$ al $65 \%$ de las demandas energéticas por vivienda, con costos del $3 \%$ al $14 \%$ de su valor. Demostrando una adecuada operación y recomendaciones para el mejoramiento habitacional, que permiten ofrecer un apoyo profesional y un medio de concientización ambiental.
\end{abstract}

PALABRAS CLAVES: Vivienda, Reacondicionamiento, Simulación de Desempeños, Divulgación Pública

\begin{abstract}
This paper presents a computational prototype for the definition and divulgation of environmental improvements of houses in the metropolitan area of Concepción, Chile. The homes generate high energy consumptions, so many public programs and private initiatives allow retrofitting existing buildings to improve their performance, with specific solutions that can be determined by dynamic simulations. It proposes a professional tool to review alternatives, including price calculations and simulation results to define packages of improvements per case. Also, this software estimates possibilities of reconditioning using basic data, and knowledge from previously studied homes, providing public awareness. The

\footnotetext{
${ }^{1}$ Depto. Diseño y Teoría de la Arquitectura, Universidad del Bío-Bío, Avda. Collao 1202, Concepción, Chile, rgarcia@ubiobio.cl

2 Depto. Sistemas de Información, Universidad del Bío-Bío, Avda. Collao 1202, Concepción, Chile, pgcampos@ubiobio.cl
} 
background and studied cases are exposed, as well as the development and procedure proposed, and some cases analyzed. The software determines housing refurbishment packages that generate reductions of $35 \%$ to $65 \%$ of its energy needs, with costs from $3 \%$ to $14 \%$ of its value. Demonstrating proper operation and relevant recommendations for housing improvements that can offer professional support and a means of residential environmental awareness.

KEYWORDS: Housing, Retrofit, Building-Performance Simulation, Public Dissemination

\section{Introducción}

El agotamiento de los recursos naturales y los problemas ambientales, están motivando acciones para reducir el consumo energético y promover una cultura más sustentable (CIB, 2013). Las viviendas albergan gran parte de las actividades humanas y requieren servicios de iluminación, equipamiento, calentamiento de agua, cocina y climatización, que consumen cerca del cuarto de la energía global (CNE, 2009). Además poseen en general un bajo confort interior y relevantes gastos, debido a condiciones constructivas débiles para su desempeño térmico, especialmente en los países en desarrollo (AC, 2007). La edificación habitacional en Chile, por ejemplo, presenta un consumo energético medio anual de aprox. $13.500 \mathrm{kWh}$ por vivienda, mayormente en calefacción y proveniente de fuentes fósiles (CDT, 2010).

Los programas de simulación ambiental permiten modelar la geometría, materialidad y ocupación de los edificios, con el fin de revisar el comportamiento de diversas condiciones interiores. Estas simulaciones permiten determinar periodos de confort o disconfort, y la energía para mantenerlos, así como la transferida desde o hacia el entorno. En los modelos se pueden cambiar características (por ejemplo, espesor de un material) y revisar la demanda energética subsecuente (HENSEN-LAMBERT, 2011). Sin embargo se carece de procedimientos para registrar los resultados y compararlos, como tampoco se dispone de alternativas para definir mejoramientos. Algunos especialistas sugieren adecuaciones para distintas tipologías o climas, pero sin detallarlas (BUSTAMANTE, 2009; ESAN 2012). Los analistas suelen realizar modelos variando condiciones, pero sin aclarar las diferencias o costos (JANKOVIC, 2012; ANDERSON, 2014). 
Por otro lado, se están incorporando conceptos de construcción sustentable en la formación, acciones estatales y campañas públicas (DOSAL, 2013). En Chile, a partir del año 2007 se han establecido regulaciones en las viviendas para incrementar su desempeño térmico, y se han establecido programas de reacondicionamiento habitacional. Sin embargo, estas acciones adolecen de procedimientos de análisis o recomendaciones específicas, revelando una escasa verificación (CDT, 2010). Por lo que la revisión de condiciones habitacionales y difusión de posibilidades de mejoramiento podría apoyar estas acciones.

El objetivo de este trabajo es desarrollar una plataforma computacional para analizar resultados de simulación ambiental de viviendas y proponer paquetes de mejoramiento, además de la estimación de casos para su difusión pública. Basado en estudios generales y el análisis de diez viviendas unifamiliares en Concepción, Chile. Con el fin de proveer un apoyo profesional y promover reacondicionamientos residenciales que ayuden a mitigar los impactos ambientales y gastos domésticos. Se expone a continuación la situación habitacional en la zona, la estrategia de análisis y un prototipo computacional, además de experiencias de prueba para verificar la herramienta y estimaciones generadas.

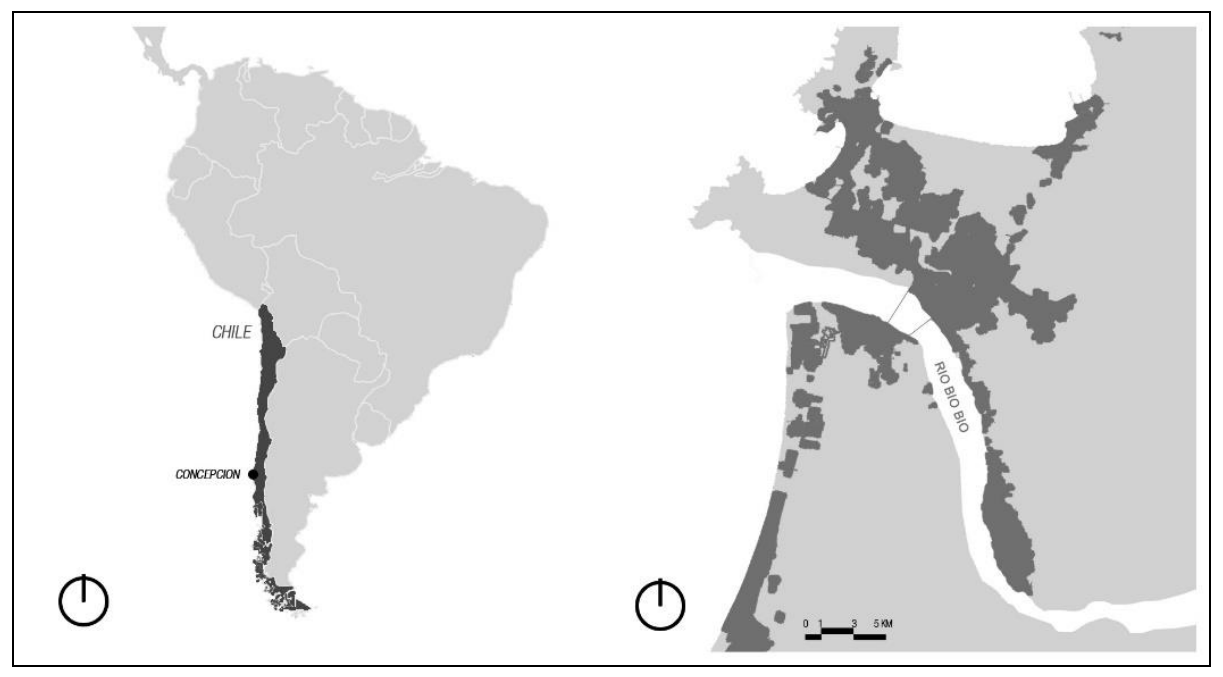

Fig.1 Localización de Concepción.

\section{Viviendas en Concepción.}


La ciudad de Concepción es el mayor área urbana del sur de Chile, con cerca de un millón de habitantes que residen en aprox. 250.000 viviendas, cerca del $80 \%$ son construcciones unifamiliares de uno o dos pisos, mayormente ejecutadas en albañilería reforzada con techumbre de madera o metal revestidos. La ciudad está localizada en $36^{\circ}$ Latitud Sur y $73^{\circ}$ Longitud Oeste, junto al Océano Pacífico y el rio Bío-Bío (Fig. 1), posee un clima templado marítimo, con una temperatura media en verano de $18^{\circ} \mathrm{C}$ y en invierno de $10^{\circ} \mathrm{C}$.

Para este trabajo, primeramente se registraron diez viviendas ubicadas en diferentes sectores de la ciudad, y que acomodan grupos familiares de distinta composición y nivel de ingresos. Seleccionadas de una convocatoria voluntaria a estudiantes universitarios. Se revisaron inicialmente cincuenta casas, y luego se eligieron diez representativas y disponibles para el estudio, que expresan diversos tamaños (de 60 a $200 \mathrm{~m}^{2}$ ), antigüedad constructiva (desde 60 a 2 años), orientación solar y configuración (Fig. 2).

Las viviendas fueron sometidas a un monitoreo de condiciones ambientales en dos semanas (una en verano y otra en invierno), con sensores de temperatura exterior, interior superficial y aérea en cuatro recintos, humedad, iluminación, nivel de $\mathrm{CO}_{2}$, hermeticidad al aire y variación térmica. Detectando una condición similar a estudios nacionales (AC, 2007; CDT, 2010), con confort interior bajo y variable según el clima, producto de alta permeabilidad y transmitancia de la envolvente. La ocupación se revisó con registros de permanencia, encuestas de satisfacción térmica, entrevistas en profundidad, observación participante y visitas intensivas. Las cuales reflejaron una aceptación de las condiciones habitacionales, con diversidades generacionales y estacionales, y entre los niveles y orientación de la vivienda. Presentaban un gasto del 5\% del ingreso familiar destinado a servicios domésticos, principalmente de consumo energético, con un incremento en invierno para calefacción, especialmente en las familias jóvenes. 


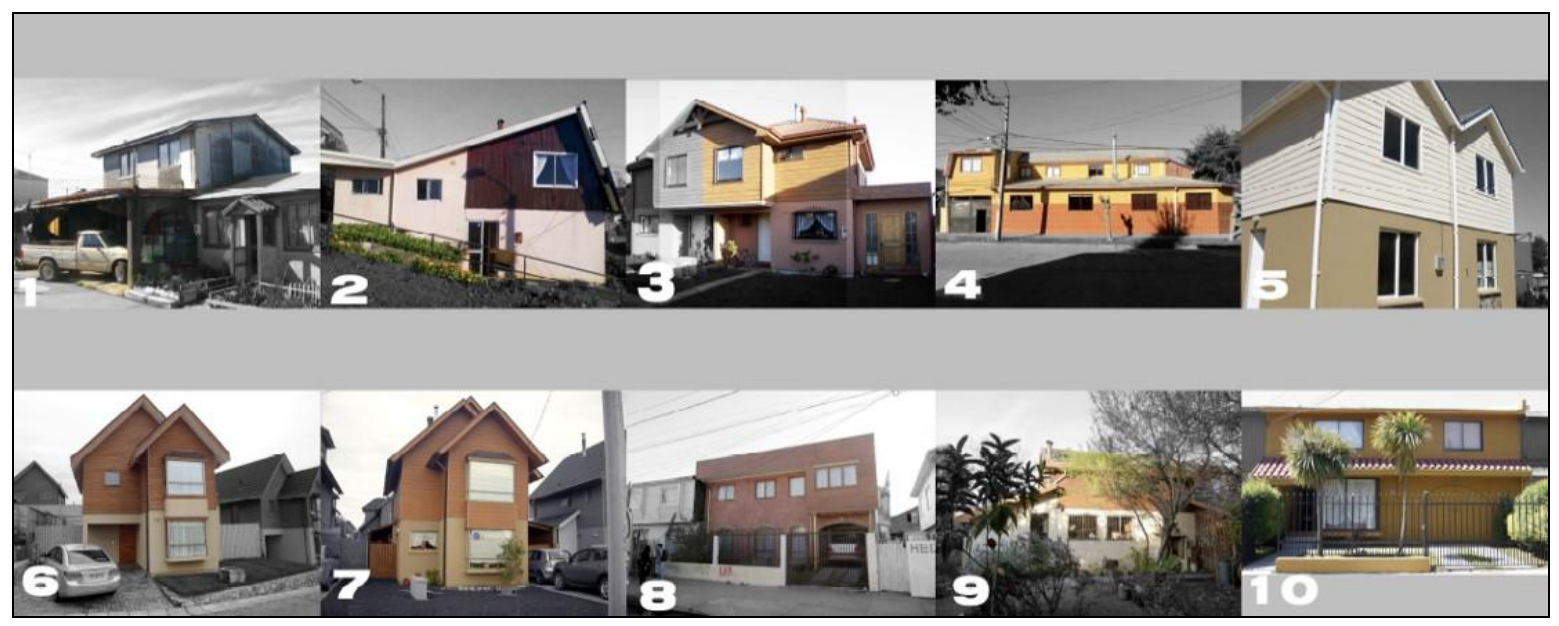

Fig.2. Fotos de Viviendas Estudiadas

Se verificaron además condiciones constructivas, climáticas, modelación geométrica, ocupación y equipamiento, y en particular renovación de aire y banda de confort, para realizar simulaciones que expresaran un comportamiento equivalente al existente. Determinando que la demanda energética de Mayo a Octubre en calefacción con un confort mínimo de $18^{\circ}$ reflejaba los gastos de las viviendas medias. Utilizando software Ecotect, Design Builder y Open Studio (con EnergyPlus), acuerdo a las normas ISO 13790:2008-09 y ASRHAE 90.1

Según recomendaciones de estudios nacionales (AC, 2007; CDT, 2010), además de simulaciones de prueba, se determinó un repertorio de adecuaciones constructivas destinadas a reducir la demanda energética y mantener el confort (POLLY et al, 2011). Destinadas a mejorar la hermeticidad y aislación, mediante la eliminación filtraciones de aire, recubrimientos adicionales en primer piso o segundo piso y ventanas con doble vidrio. Otras alternativas, como ampliación o eliminación de ventanas, invernaderos, tragaluces, ventilaciones, aislación de pisos, etc., no revelaron aportes importantes.

En los casos estudiados se advirtieron regularidades en las demandas energéticas y soluciones constructivas, que permiten sugerir posibilidades generales, como también definiciones precisas según resultados de simulaciones. Para lo cual se planteó un procedimiento de análisis y proyecciones en una herramienta computacional, mediante el uso del paradigma de programación orientada a objetos en lenguaje Java, con el fin de 
elaborar una herramienta de uso profesional y también de divulgación pública, con características de alta mantenibilidad del software, permitiendo su modificación y extensión a futuro.

\section{Desarrollo}

La elaboración del sistema computacional, actualmente en etapa de prototipo funcional avanzado, consideró los siguientes pasos: determinar la estrategia de análisis, establecer métricas y cálculos por vivienda, establecer un proceso simplificado para estimaciones, definir un diseño del software flexible y escalable, y la interfaz de usuario para ambas modalidades.

\subsection{Análisis por vivienda}

Las actividades consideradas para generar paquetes de mejoramiento térmico residencial son las siguientes (ver Fig. 3):

1) Datos iniciales: consiste en registrar información constructiva de la vivienda que es relevante para evaluar su desempeño térmico, tales como superficie, cantidad de puertas, cantidad de ventanas, etc.

2) Simulación base: desarrollo de un modelo ambiental de cada vivienda, mediante software de simulación dinámica, lo que permite obtener demandas energéticas de la vivienda para un confort adecuado, y en particular, requerimientos de calefacción.

3) Simulación de Alternativas: modelación de la vivienda con diferentes modificaciones destinadas a reducir su consumo energético (de aquí en adelante llamadas soluciones). Cada solución se simula de forma independiente, con el objeto de obtener resultados de demanda con cada alternativa.

4) Análisis Económico: consiste en una evaluación costo vs. beneficio de cada solución, a fin de determinar su eficiencia. Para esto, se determina el monto de ejecución de cada alternativa, considerando el precio de materiales y mano de obra y las cantidades requeridas en base a la información sobre geometría de la vivienda. Finalmente, se calcula 
la eficiencia de la solución, en términos del costo de cada $\mathrm{kWh}$ ahorrado, de acuerdo a la ecuación (1).

$$
\text { Eficiencia }=\frac{\text { Costo de la solución }[\$]}{\text { Ahorro en demanda energética }[\mathrm{kWh}]}
$$

5) Generación de paquetes: agrupamiento de las soluciones en orden de eficiencia, sumando su costo acumulado hasta un monto inferior al 5\%, 10\% o $15 \%$ del valor comercial de la vivienda, con el fin de ofrecer distintas magnitudes de inversión. El monto del $10 \%$ equivale al subsidio estatal para estas acciones. Además se excluyen soluciones incompatibles (por ejemplo, si se incluye el sellado y el cambio de ventanas en un mismo paquete, se elimina la solución menos eficiente).

6) Consulta: los paquetes generados son presentados y explicados a profesionales, ejecutores o habitantes de la vivienda, para que tomen la decisión de inversión e implementen el paquete de soluciones seleccionado.

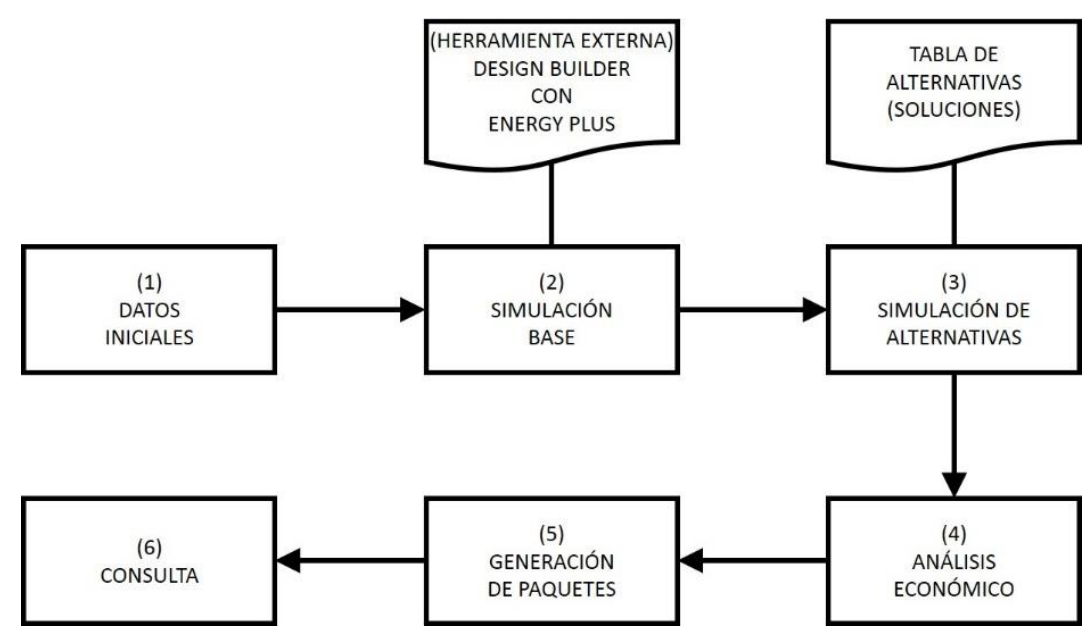

Figura 3: Metodología para la generación de paquetes de reacondicionamiento energético.

\subsection{Automatización del proceso}


La estrategia se desarrolló inicialmente con planilla de cálculo para determinar los costos de las soluciones, registrar resultados de simulaciones y generar los paquetes. Lo cual implica una preparación y variedad de tareas manuales. Debido a lo anterior, se estableció una automatización del proceso en tres módulos principales. El primer módulo, se encarga de la recopilación de los datos de la vivienda para cuantificar las soluciones. El segundo módulo, se encarga de realizar el análisis de las alternativas. El tercer módulo, se encarga de la construcción de paquetes de soluciones. Estos módulos son descritos a continuación.

\section{a) Recolección de datos}

Corresponde al módulo que recoge características de cada vivienda para determinar los costos de cada solución. Primeramente, datos de identificación y tasación comercial (su valor estimado de venta,), y el conjunto de métricas para cuantificar las soluciones de reacondicionamiento térmico (Tabla 1 ).

\begin{tabular}{|l|l|}
\hline Nombre & Descripción \\
\hline Puertas & Cantidad de puertas al exterior de la vivienda \\
\hline Ventanas & Cantidad de ventanas de la vivienda \\
\hline Perforaciones & $\begin{array}{l}\text { Cantidad de aperturas adicionales en muros debido a } \\
\text { conductos eléctricos, tuberías de aguas, uniones, etc. }\end{array}$ \\
\hline Guardapolvos & Longitud de bordes de muros exteriores, en $\mathrm{ml}$ \\
\hline Pisos & Cantidad de niveles de altura de la vivienda \\
\hline Superficie periferia piso 1 & Extensión del muro exterior del primer piso, en $\mathrm{m}^{2}$ \\
\hline Superficie periferia piso 2 & Extensión del muro exterior del segundo piso, en $\mathrm{m}^{2}$ \\
\hline Superficie ventanas & Sumatoria del área de ventanas de la vivienda, en $\mathrm{m}^{2}$ \\
\hline Superficie cielo & Superficie total interior de la techumbre, en $\mathrm{m}^{2}$ \\
\hline
\end{tabular}

Tabla 1: Métricas de vivienda usadas para cálculo de soluciones.

Además se registra el resultado de demanda energética de calefacción en invierno de la vivienda, en $\mathrm{kWh}$, según la simulación de comportamiento térmico con las condiciones normalizadas (correspondiente al paso 2).

\section{b) Análisis de soluciones constructivas}

El módulo para cálculos de las diferentes alternativas de mejoramiento energético, registra su costo y resultado de demanda de una simulación con la adecuación aplicada. Las 
soluciones se basan en las recomendaciones generales para esta tipología. Considerando sellados de elementos, como puertas, ventanas o perforaciones en muros y la aplicación de aislamientos exteriores en muros (tipo EIFS: Exterior Insulation and Finish System) de primer o segundo piso, en cielos de los recintos o ventanas con doble vidrio (Tabla 2).

\begin{tabular}{|c|l|l|}
\hline Identificador & Nombre & Descripción \\
\hline 1 & Sellado de puertas & $\begin{array}{l}\text { Colocar cintas en ranuras laterales de puertas que dan al } \\
\text { exterior de la vivienda }\end{array}$ \\
\hline 2 & Sellado de ventanas & Colocar cintas en bordes de ventanas exteriores \\
\hline 3 & $\begin{array}{l}\text { Sellado de } \\
\text { perforaciones }\end{array}$ & $\begin{array}{l}\text { Colocación de sellos laterales en aberturas de cableado } \\
\text { eléctrico, tuberías sanitarias y de ventilación, etc. }\end{array}$ \\
\hline 4 & $\begin{array}{l}\text { Sellado de perímetro } \\
\text { de muros }\end{array}$ & $\begin{array}{l}\text { Adhesivos en bordes inferiores y superiores de muros } \\
\text { exteriores (guardapolvos y cornisas) }\end{array}$ \\
\hline 5 & $\begin{array}{l}\text { Aislamiento en } \\
\text { primer piso }\end{array}$ & $\begin{array}{l}\text { Revestimiento adicional aislado (EIFS) en muros exteriores del } \\
\text { primer nivel de la vivienda }\end{array}$ \\
\hline 6 & $\begin{array}{l}\text { Aislamiento segundo } \\
\text { piso }\end{array}$ & $\begin{array}{l}\text { Revestimiento adicional aislado (EIFS) en muros exteriores del } \\
\text { segundo nivel de la vivienda }\end{array}$ \\
\hline 7 & Cambio de ventanas & $\begin{array}{l}\text { Remplazo de ventanas exteriores con marcos con doble sello y } \\
\text { doble vidrio hermético (DVH) }\end{array}$ \\
\hline 8 & Aislamiento cielos & $\begin{array}{l}\text { Aplicación de aislamiento adicional en cielos sobre los recintos } \\
\text { superiores }\end{array}$ \\
\hline
\end{tabular}

Tabla 2. Lista de soluciones de mejoramiento energético

Según las métricas, se calcula luego la cantidad de solución por vivienda, y después se multiplica por el precio unitario, para determinar el costo total de cada adecuación. Luego, según la demanda de la simulación, se determina el eventual ahorro energético (paso 3). Con lo que se calcula la eficiencia de cada solución, de acuerdo a la ec. (1), correspondiente al paso 4 de la estrategia.

\section{c) Generación de paquetes:}

Corresponde al módulo de agrupación de soluciones, con el fin de disponer de diferentes alternativas de gasto, jerarquizando las soluciones en magnitud y rentabilidad (cuales generan más ahorro total y son más efectivas). Para ello, primero se ordenan las soluciones en base a su eficiencia. Luego, se agregan soluciones en cada paquete hasta aproximarse al monto global. En caso de incluir soluciones que son incompatibles entre sí, se elige aquella que produzca un mayor ahorro de demanda energética, de acuerdo al paso 5 de la estrategia. El procedimiento implementado se describe en la Fig. 4: 
Revista Tecnologia e Ambiente, v. 21, n.1, 2015, Criciúma, Santa Catarina. ISSN: 1413-8131 (versão impressa) ISSN: 2358-9426 (versão eletrônica)

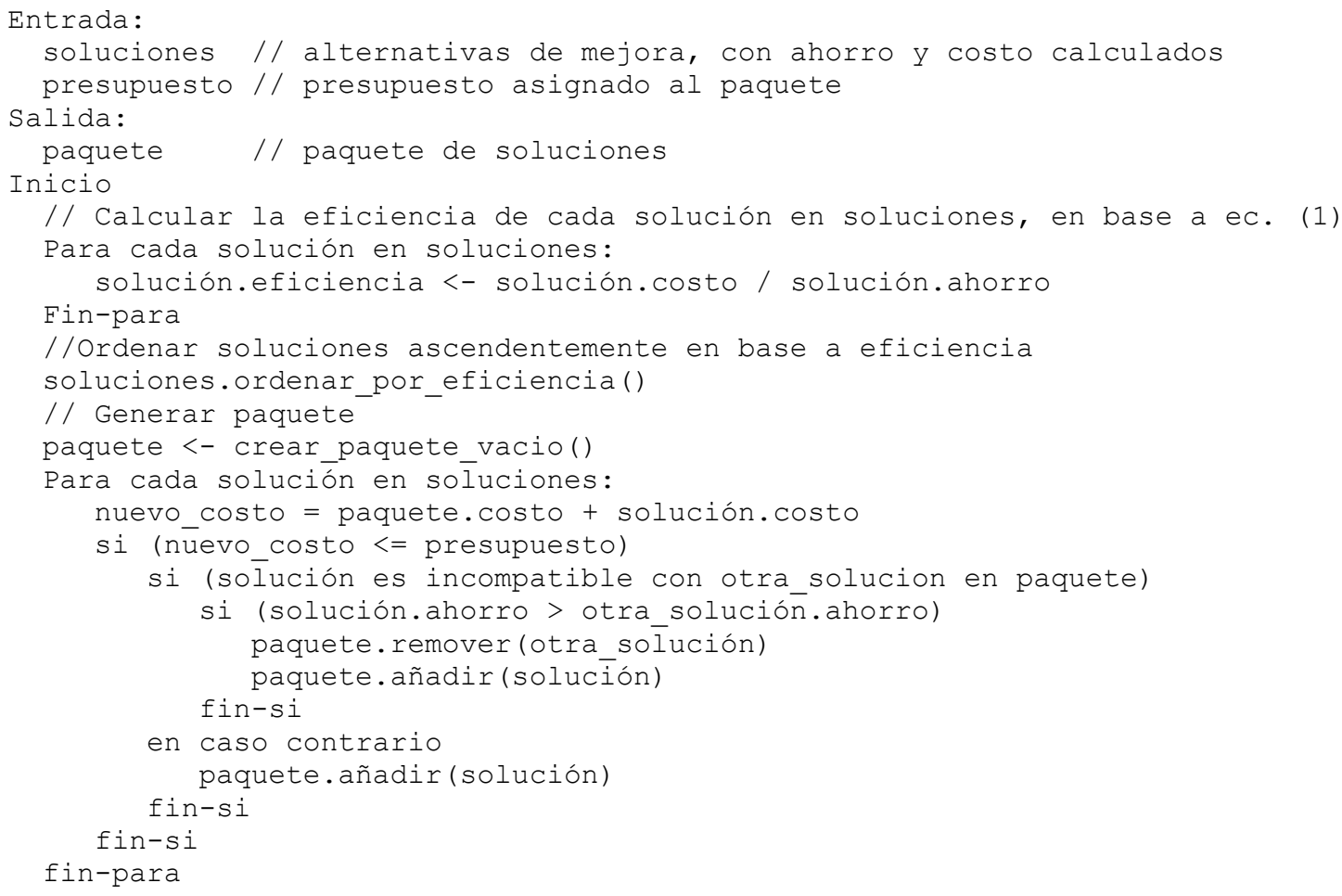

Figura 4: Procedimiento de generación de paquetes en el prototipo.

Finalmente, la descripción de los paquetes generados, en términos de las soluciones incluidas, su costo, eficiencia y ahorro en demanda energética, es presentada a los usuarios del software, correspondiendo al último paso de la estrategia.

\subsection{Procedimiento de Estimaciones}

Como una forma de otorgar sugerencias preliminares de rehabilitación para público general, sin la necesidad de efectuar simulaciones, se consideró el funcionamiento adicional del prototipo con valores proyectados de los casos previamente estudiados, reduciendo de este modo los datos y tareas requeridas. Las magnitudes estimadas se pueden fortalecer después, con mayor cantidad de viviendas simuladas y acondicionamientos ejecutados. El proceso de recomendación de adecuaciones solicita algunos datos para estimar la demanda base y cuantificar las soluciones (algunas similares al proceso con simulación, pero sin registro de precios o resultados). Conformando un conjunto reducido de métricas (Tabla 3). 


\begin{tabular}{|l|l|}
\hline Nombre & Descripción \\
\hline Superficie & $\begin{array}{l}\text { Área total de los recintos habitables de la vivienda, en } \mathrm{m}^{2} \text {. Se requiere } \\
\text { para estimar demanda energética de calefacción de la vivienda que } \\
\text { depende fundamentalmente del tamaño de los espacios. }\end{array}$ \\
\hline Año Construcción & $\begin{array}{l}\text { Año de ejecución inicial de la vivienda. Se requiere para ajustar la } \\
\text { estimación de demanda energética, pues las normas de construcción } \\
\text { habitacional a partir del año 2007 redujeron las demandas. }\end{array}$ \\
\hline Perímetro expuesto & $\begin{array}{l}\text { Longitud de los muros exteriores de la vivienda, en m. Se requiere } \\
\text { para cuantificar soluciones de revestimiento y sellado de paramentos. }\end{array}$ \\
\hline Cantidad de pisos & $\begin{array}{l}\text { Indicación respecto de si la vivienda cuenta con 1 o } 2 \text { niveles, para } \\
\text { estimar alturas generales y variedad de soluciones. }\end{array}$ \\
\hline Puertas & $\begin{array}{l}\text { Cantidad de puertas exteriores para estimar soluciones sobre puertas } \\
\text { existentes. }\end{array}$ \\
\hline Ventanas & $\begin{array}{l}\text { Cantidad de ventanas exteriores de la vivienda, para estimar } \\
\text { soluciones aplicadas sobre vanos. }\end{array}$ \\
\hline
\end{tabular}

Tabla 3: Métricas reducidas de vivienda para estimación de soluciones.

Para estimar las soluciones (y excluir la consulta de datos técnicos de difícil comprensión al público), se considera un conjunto de supuestos (Tabla 4).

\begin{tabular}{|l|l|}
\hline Métrica Básica & Forma de estimación \\
\hline Perforaciones & Superficie total $\times 0,4$ \\
\hline Guardapolvos & Perímetro expuesto $\times$ Cantidad pisos $\times 2$ \\
\hline Superficie periferia piso 1 & Perímetro expuesto $\times 2$ \\
\hline Superficie periferia piso 2 & Perímetro expuesto $\times 2$ (sólo si la vivienda tiene 2 pisos) \\
\hline Superficie ventanas & Ventanas $\times 1,2 \mathrm{~m}^{2}$ \\
\hline Superficie cielo & Superficie $/ 2$ (si tiene dos pisos) \\
\hline
\end{tabular}

Tabla 4: Estimación de métricas básicas

Para la estimación de la demanda energética, a partir del análisis de los casos de estudio y simulaciones realizadas, se observó una alta correlación negativa entre tamaño de vivienda (superficie) y resultado total de calefacción. La cual se ve influenciada además por el año de ejecución, debido a la introducción de normas más eficientes de construcción el año 2007. Al separar los datos de viviendas construidas antes del 2007 se obtuvo un coeficiente de correlación de Pearson de $r=-0.7324$, mientras que para los datos de las restantes viviendas se obtuvo un coeficiente $r=-0.9221$. Así, se calcularon regresiones lineales a partir de los datos existentes, obteniéndose la ec. (2) para el cálculo de demanda energética por metro cuadrado en viviendas previas al 2007, y la ec. (3) para viviendas posteriores: 


$$
\begin{gathered}
\text { Demanda por } m^{2}(\text { previa a } 2007)=-0,4654 \times \text { superficie en } m^{2}+169,79 \\
\text { Demanda por } m^{2}(2007 \text { en adelante })=-0,3599 \times \text { superficie en } m^{2}+104,61
\end{gathered}
$$

Cabe señalar que para grandes superficies se utiliza un valor constante, asumiendo una estabilización en la demanda energética sobre un determinado límite (debido a que las viviendas mayores usualmente no poseen más habitantes, por lo que no generan un mayor requerimiento energético).

Por otro lado, también del análisis de los casos y simulaciones realizadas, se considera una reducción estimada de la demanda con cada solución. Los precios unitarios se establecieron en base a revisión de costos medios de mercado. El detalle de los precios y reducciones de demanda contemplados en la versión actual se muestran en la Tabla 5.

\begin{tabular}{|c|l|r|r|}
\hline Identificador & Nombre & $\begin{array}{l}\text { Precio } \\
\text { Unitario }\end{array}$ & $\begin{array}{l}\text { Reducción total en } \\
\text { demanda energética }\end{array}$ \\
\hline 1 & Sellado de puertas & US\$ 19 & $10 \%$ \\
\hline 2 & Sellado de ventanas & US\$ 12 & $5 \%$ \\
\hline 3 & Sellado de perforaciones & US\$ 13 & $10 \%$ \\
\hline 4 & Sellado de perímetro de muros & US\$ 9 & $20 \%$ \\
\hline 5 & Aislamiento primer piso & US\$ 55 & $10 \%$ \\
\hline 6 & Aislamiento segundo piso & US\$ 261 & $10 \%$ \\
\hline 7 & Cambio de ventanas & US\$ 16 & $5 \%$ \\
\hline 8 & Aislamiento cielos & US & \\
\hline
\end{tabular}

Tabla 5: Precio y reducción de demanda asociados a soluciones de mejoramiento energético.

El cálculo de demandas fue refinado para la solución 2 (sellado de ventanas), pues se observó que en viviendas de gran tamaño, el impacto de esta solución es menor, debido al tipo de ventana. Por lo anterior, se añadió una regla específica que rebaja la estimación de ahorro de demanda energética al $5 \%$ en viviendas de superficie sobre $200 \mathrm{~m}^{2}$.

\subsection{Diseño detallado de software}

A partir de la estrategia de análisis, automatización y estimación revisada en las secciones anteriores, se realizó un diseño de software que permitiese una programación 
eficiente y flexible, para posteriores mejoras y extensiones en la funcionalidad (KOZLOV et al., 2008).

En base a los requisitos de mantenibilidad y escalabilidad, se escogió el paradigma de orientación a objetos (RUMBAUGH et al., 1991), por sus características de programación modular, bajo acoplamiento y alta cohesión en los módulos programados. El lenguaje seleccionado fue Java (ARNOLD et al., 1996), por su alta portabilidad (puede ser ejecutado en diferentes computadores y sistemas operativos). El módulo de recolección de datos fue implementado como una aplicación de escritorio, utilizando el toolkit Swing provisto por Java (ECKSTEIN et al., 1998), mientras que el módulo de cálculo de alternativas de mejoramiento fue implementado como una librería de clases, trabajando en conjunto con el módulo de generación de paquetes, según muestra la Fig. 5.
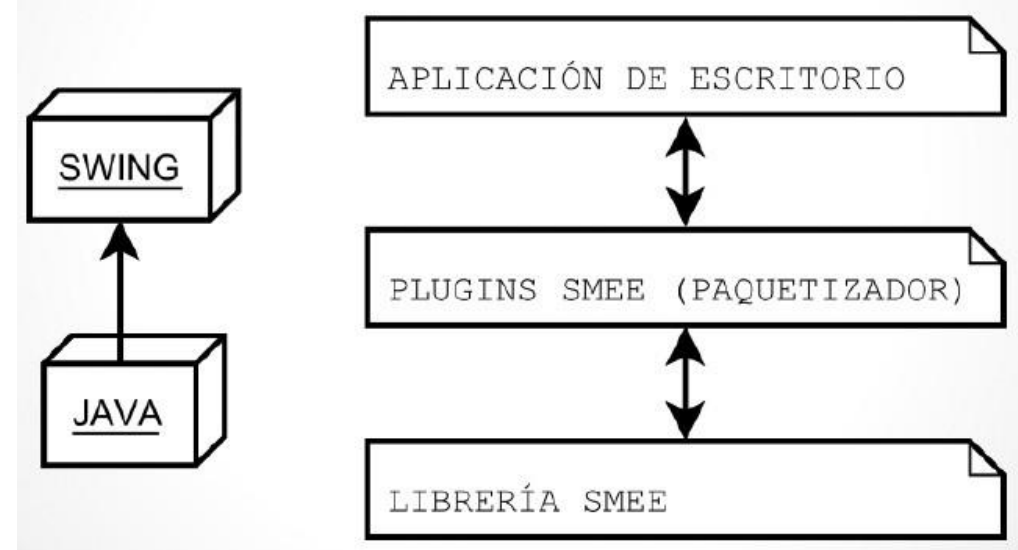

Figura 5: Tecnologías y módulos implementados en la herramienta software.

Finalmente, la Fig. 6 muestra las principales clases de objetos de software que contiene la librería que implementa el módulo de cálculo de alternativas. Este diseño se basa en los conceptos del proceso de análisis, con el fin de facilitar la comprensión del diseño y futuras modificaciones (LARMAN, 2005). En la Fig. 6, se puede apreciar que cada paquete contiene un conjunto de soluciones específicas para una vivienda, descrita por métricas, siendo las soluciones específicas identificadas a partir del conjunto de soluciones genéricas disponibles en la librería, algunas de las cuales son incompatibles entre sí. 


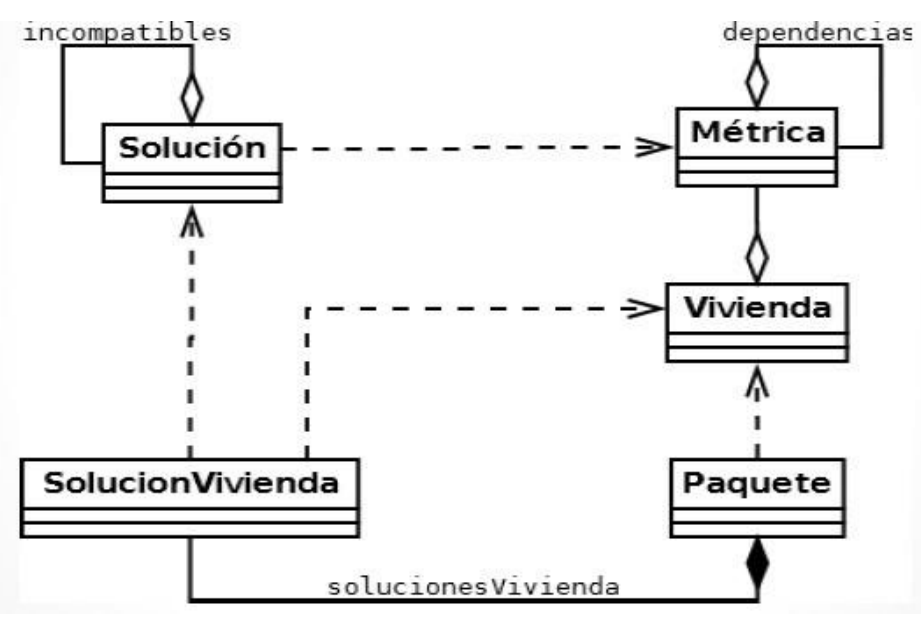

Figura 6: Clases implementadas en la herramienta software.

\subsection{Diseño de Interfaz de Usuario}

Para un proceso adecuado con el usuario, en el prototipo considera un recuadro inicial, que solicita los datos de identificación de la vivienda, y otorga la opción de estimar soluciones. Si la opción no es activada, se deriva al proceso de análisis (Fig.7 a la derecha), en que se despliega primero un recuadro para requerir las métricas de las soluciones, y luego otro para anotar los precios unitarios y valor de demanda de calefacción en invierno otorgada por la simulación base, y los resultados consecuentes de simular con las distintas alternativas. Por otro lado, en la opción de estimar (Fig.7 hacia abajo) se presenta un recuadro que solicita los datos reducidos para cuantificar demandas y costos de las soluciones, luego se generan los paquetes, presentando las métricas estimadas. Ambas modalidades concluyen con un recuadro que despliega un menú lateral con los tres paquetes y en cada uno de éstos, se detallan la demanda total estimada y su costo, el porcentaje de ahorro energético y el costo aproximado de las soluciones. Más abajo se indica cada solución considerada, como también una evaluación financiera y gráficos comparativos de los paquetes. 


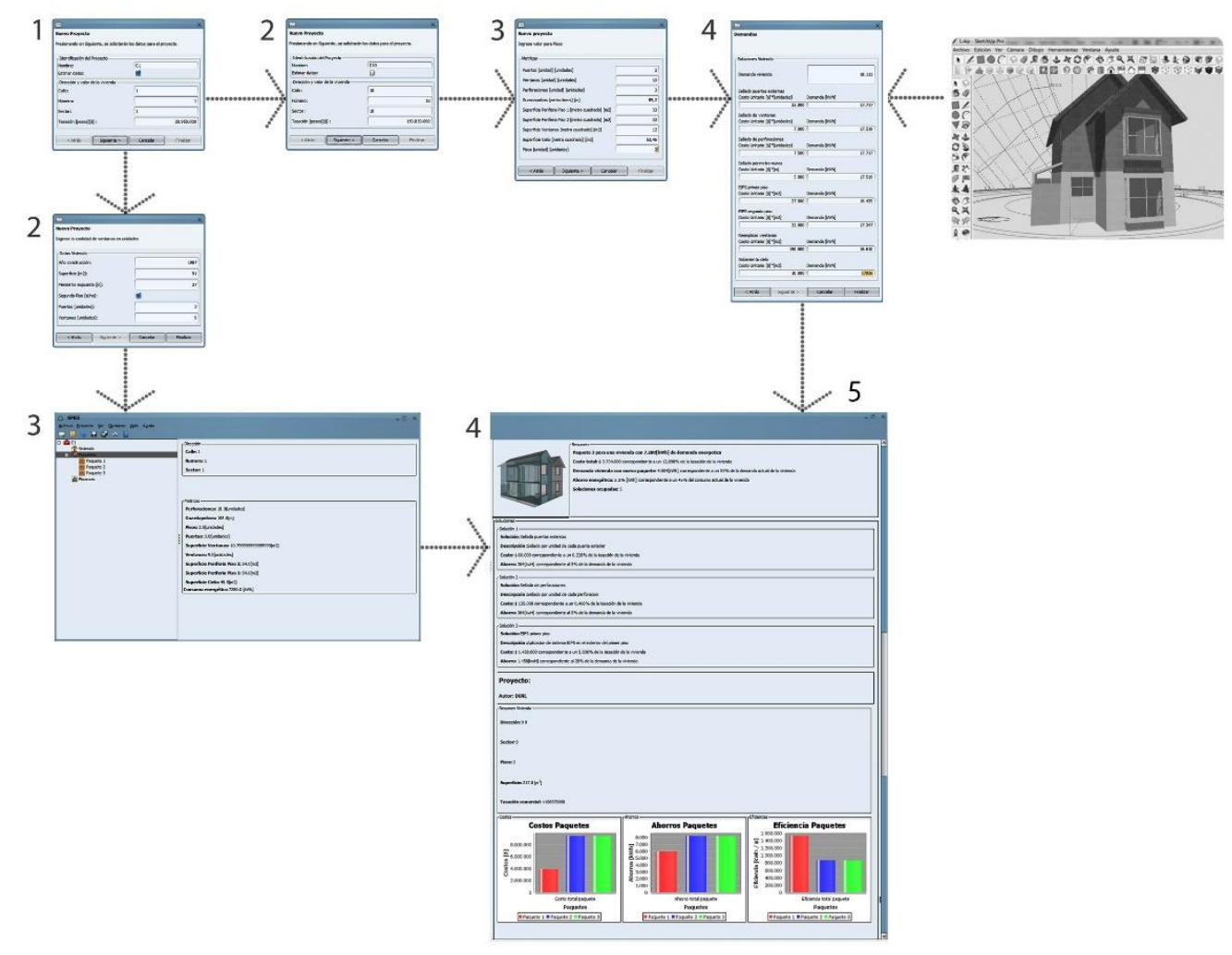

Fig.7. Ventanas del prototipo en secuencias de; estimación (hacia abajo) o análisis (a la derecha).

\section{Análisis de Casos}

Para revisar la utilización del sistema desarrollado se efectuaron pruebas comparativas en seis casos de las viviendas estudiadas. Los seis casos de estudio son construcciones individuales de dos pisos, en albañilería reforzada en primer piso y estructura de madera en segundo piso y techumbre, que alojan a familias de tres a seis integrantes. Se determinaron paquetes de mejoramiento, por un lado mediante simulaciones y cálculo individual, y por otro, realizando estimaciones con el prototipo. En ambos procesos con usuarios profesionales independientes. Lo que permitió verificar los procedimientos y la usabilidad general del programa. Los usuarios fueron seis arquitectos con un conocimiento de métodos de simulación y eficiencia energética (cuatro con formación de postgrado) y todos con experiencia de más de seis proyectos desarrollados. Después de utilizar el prototipo, respondieron una consulta escrita, en que indicaron que 
consideraron el sistema comprensible y de utilidad para profesionales en la modalidad de análisis, pero especialmente para público general en las recomendaciones. Valoraron su funcionamiento, información y presentación, aunque plantearon varias sugerencias sobre la gráfica, edición de datos, asociación de programas y precisión de información.

En la definición de paquetes por simulación, fue desarrollada con distintos profesionales, que modelaron cada vivienda, y luego efectuaron variaciones con cada solución constructiva, del cual se extrajo el valor de demanda total de calefacción. Luego en una planilla se registraron los montos, para cuantificar cada solución y después calcular su eficiencia según la energía ahorrada. Se ordenaron manualmente las soluciones de acuerdo a su efectividad, generando montos acumulados y de acuerdo al máximo posible para cada paquete, se estableció el conjunto de soluciones. Este procedimiento, disponiendo de la base de precios y modelación de cada vivienda, demoró unas seis horas por caso, con algunos errores frecuentes que exigen verificación.

Por otro lado, los profesionales que utilizaron el prototipo, revisaron documentación gráfica de cada caso (fotografías y planos), completando los datos básicos y generando los paquetes en aproximadamente diez minutos por vivienda. Con ambos procedimientos se determinaron mejoramientos constructivos que plantean reducciones del $35 \%$ al $65 \%$ de los consumos energéticos de las viviendas (Fig. 8), con costos del 3\% a 14\%. En general las propuestas con los dos procesos privilegian sellos de puertas y aislación adicional de muros en primer piso, en segunda o tercera instancia consideran cambiar ventanas y revestimiento de segundo piso.

Los resultados de demanda energética entre las simulaciones y las estimaciones del prototipo son bastante próximos, del 3\% al 15\%, pero se distancian si las viviendas son de mayor tamaño y antigüedad. Los ahorros por paquete mantienen también una magnitud y escalamiento aproximado en cada vivienda, pero también se distancian en las mayores. De manera similar en los costos, aunque en este aspecto las diferencias se reducen. 


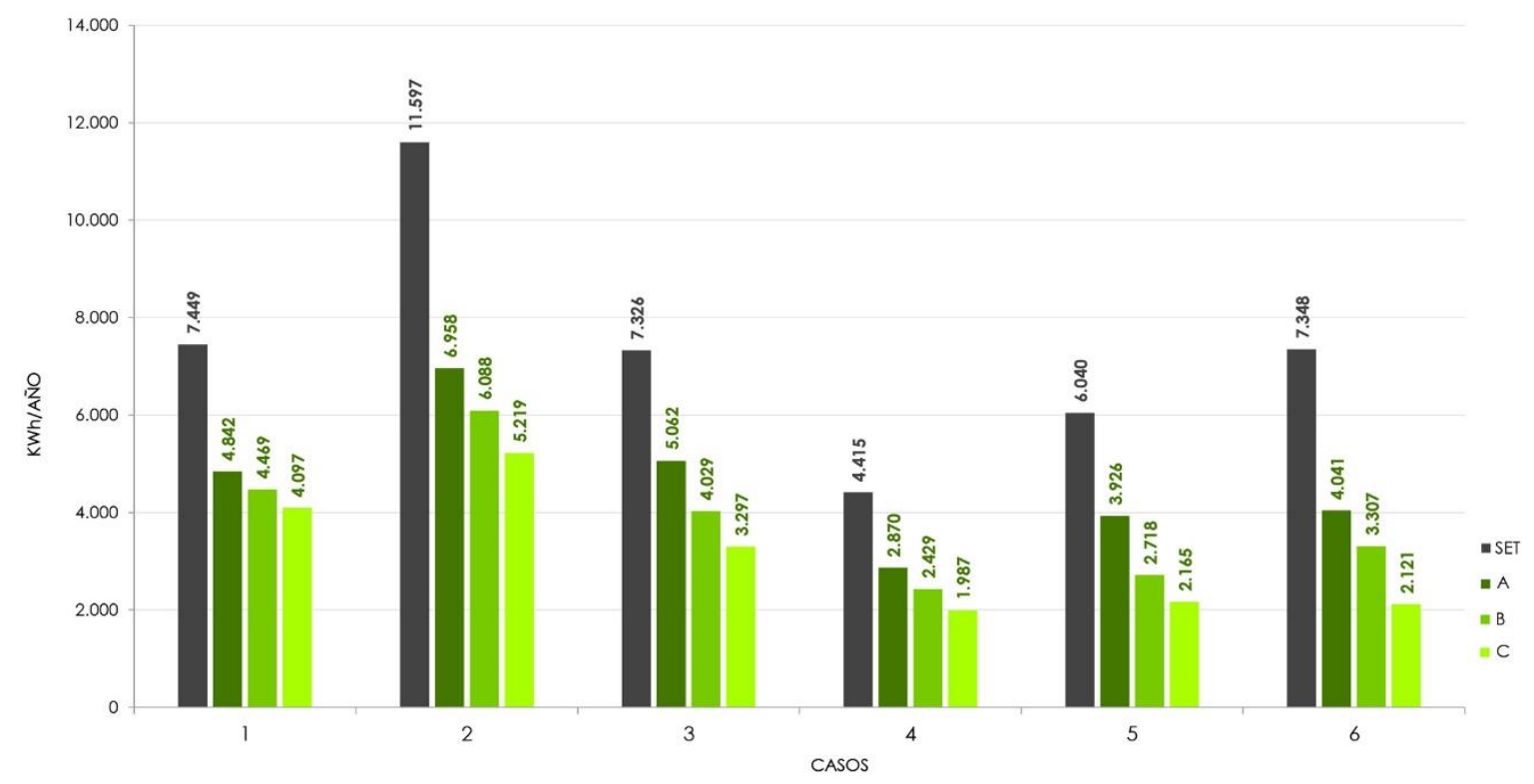

Fig.8. Gráfico de Demandas por Paquete estimado en cada Caso

\section{Conclusiones}

Este trabajo expone una implementación computacional para apoyar reacondicionamientos de viviendas existentes destinados a reducir su demanda energética y asegurar su confort interior, en construcciones habitacionales unifamiliares de la ciudad de Concepción, al sur de Chile. Planteando una herramienta de análisis y estimación de paquetes de mejoramiento, basadas en el estudio de casos y simulaciones dinámicas, además de antecedentes generales y revisión de condiciones constructivas. Elaborando un prototipo de software programado en Java, escalable y editable, que otorga funcionalidades de cálculo según resultados de simulación, o proyecciones basadas en experiencias previas y tendencias de los casos revisados. Finalmente se indica el análisis de seis viviendas mediante simulaciones y el prototipo desarrollado, aplicado por profesionales experimentados, que demuestran una utilización adecuada y con menos requerimientos personales e informáticos.

Este desarrollo plantea primeramente una herramienta profesional que permite aplicar y reducir el trabajo de simulación de modelos de viviendas para la definición de 
reacondicionamientos térmicos. Como también propone un sistema de uso público para estimar directamente los mejoramientos posibles con algunos datos básicos, provenientes de casos previamente estudiados. En ambos modos de utilización está limitado a la tipología constructiva y área geográfica según las viviendas originalmente analizadas, lo que es relevante por cuanto las soluciones constructivas pueden variar significativamente según la materialidad, volumetría y clima local. Las estimaciones en particular están limitadas a los supuestos, precios y ahorros considerados, por tanto la variación económica de algunos materiales, así como la eliminación o surgimiento de procedimientos constructivos, o un caso de vivienda complejo e inusual, requiere una verificación individual y/o modificación de la programación. Considerando estos alcances, igualmente el programa puede apoyar y divulgar posibilidades de mejoramiento de las viviendas usuales en la zona.

El prototipo elaborado debe ser refinado en su funcionamiento y presentación, con mayor información de las condiciones de operación, para su disponibilidad profesional y pública. La plataforma utilizada permite disponerlo a través de sistemas web y complementarlo con proyecciones financieras para las distintas soluciones (alternativas de pago según créditos y recuperación de inversión), se puede vincular con proveedores o ejecutores que precisen antecedentes técnicos u ofrezcan posibilidades de ejecución. El sistema también puede ampliar su precisión con una mayor variedad de soluciones, tipologías y zonas de trabajo, según casos estudiados. Es posible también implementar en el propio programa el registro de simulaciones o nuevas alternativas consideradas para otorgarle un aprendizaje automático y crecimiento progresivo de las proyecciones realizadas. Puede estar vinculado a cartografías urbanas (mapas), para facilitar la identificación y cálculo de dimensiones constructivas (seleccionando la vivienda, generar la estimación de mejoramientos posibles), como también implementarse en dispositivos móviles, con lectura de algunos datos de registro (por ejemplo, magnitudes de muros exteriores) para cuantificar directamente las alternativas o reconocer materialidades, y ajustar las soluciones según la calidad de los elementos constructivos. Estas posibilidades otorgan una relevante perspectiva al trabajo profesional y al conocimiento público de 
reacondicionamientos habitacionales para alcanzar las metas esperadas de reducción energética y calidad residencial.

\section{Referencias}

AC, Programa de Inversión Pública para Fomentar el Reacondicionamiento Térmico del Parque Construido de Viviendas, Santiago: Ambiente Consultores, 2007.

ANDERSON K, Design Energy Simulation for Architects, New York: Routledge, 2014. ARNOLD, K., GOSLING, J., HOLMES, D., and HOLMES, D. 1996. The Java programming language. New York: Addison-Wesley, 1996.

BUSTAMANTE, W. Guía de Diseño para la Eficiencia Energética de la Vivienda Social, Santiago: Ministerio de Vivienda y Urbanismo, 2009.

CDT, Estudio de Usos Finales y Curva de Oferta de Conservación de la Energía en el Sector Residencial, Santiago: Corporación de Desarrollo Tecnológico, 2010.

CIB, The implementation of energy efficient buildings policies: an international comparison, Paris: International Council for Research and Innovation in Building and Construction, 2013.

CNE, Antecedentes sobre la matriz energética en Chile y sus desafíos futuros, Santiago: Comisión Nacional de Energía, 2009.

DOSAL C. 2013, Eficiencia Energética y Ambiental en el Sector Vivienda, Prácticas Nacionales e Internacionales, México: Fundación Idea y Embajada Británica, 2013. ECKSTEIN, R., LOY, M., and WOOD, D. 1998. Java swing. O'Reilly \& Associates, Inc. 1998.

ESAN K. 2012, Analysis of Housing Upgrades for Policy Formulation Using Dynamic Simulation Tool, Unpublished Ph.D. dissertation, University of Stratchlyde, Glasgow, UK. HENSEN J. \& LAMBERT T. Building Performance Simulation for Design and Operation, London: Spon Press, 2011.

JANKOVIC L, Designing Zero Carbon Buildings Using Dynamic Simulation Methods, New York: Routledge, 2012. 
KOZLOV, D., KOSKINEN, J., SAKKINEN, M. and MARKKULA, J. Assessing maintainability change over multiple software releases'. J. of Software Maintenance and Evolution, 20 (1), 2008, 31-58.

LARMAN, C. Applying UML and patterns: an introduction to object-oriented analysis and design and iterative development. Pearson Education, 2005.

POLLY B. GESTWICK M. BIANCHI, M. ANDERSON R., HOROWITZ S.

CHRISTENSEN C. AND JUDKOFF R., A Method for Determining Optimal Residential Energy Efficiency Retrofit Packages, Golden: National Renewable Energy Laboratory, 2011.

RUMBAUGH, J., BLAHA, M., PREMERLANI, W., EDDY, F., and LORENSEN, W. E. Object-oriented modeling and design. Englewood Cliffs: Prentice-Hall, 1991. 